\title{
NON-DESTRUCTIVE TESTING OF CONDUCTIVE MATERIAL BY EDDY CURRENT AIR PROBE BASED ON SWEPT FREQUENCY
}

\author{
Andrea Štubendeková — Ladislav Janoušek *
}

\begin{abstract}
This article deals with non-destructive testing of conductive materials by eddy current method based on swept frequency. A 2D numerical model of eddy current air-core probe was created in the LTspice software at first. The probe consists of two coils: exciting coil and receiving. The probe is positioned in air in this case. Experimental and calculated frequency responses of the probe are obtained within a wide frequency range using swept frequency in order to validate the model. Frther a 3D model used for non-destructive testing of austenitic steel plate with three electro-discharge machined notches is then developed accordingly. Results of measurement are compared with 3D numerical model using COMSOL Multiphysics software.

K e y w ords: non-destructive testing, eddy current method, swept frequency, circuit model, air probe, numerical
\end{abstract} simulation

\section{INTRODUCTION}

Currently, modern approaches such as system health monitoring and condition based maintenance are used for evaluation of structural integrity of various elements and structures. Non-destructive testing of conductive materials by eddy current method is often employed for this purpose as it provides good results. The new approaches require continuous monitoring with fixed sensor and this is challenging issue for eddy current testing (ECT) because standard inspections require movement of the probe over the surface under the test. The authors propose a new driving technique to solve the issue. One of the possibilities is the application of the swept frequency eddy current techniques, which uses the fixed location of a probe positioned over a material while the frequency of excitation signal is changed in a wide range.

\section{EDDY CURRENT METHOD BASED ON SWEPT FREQUENCY}

Eddy currents can be used as a method for nondestructive testing, which evaluates the state of an object without damaging it. The fundamentals of the eddy current method are based on the phenomenon of electromagnetic induction. The method can thus be applied only for conductive materials. If a coil supplied with a timevarying electrical current is positioned close to the surface of an inspected object, it eventually causes flow of eddy currents in it. The shape of the current lines induced in planes perpendicular to the vector of magnetic induction $B$ is reminiscent of a whirlpool. Therefore, they are called eddy currents. Their flow in a tested material is influenced by the material properties, namely electrical conductivity $\gamma$, magnetic permeability $\mu$, the presence of discontinuities in the material, dimensions of the material and the excitation frequency. The eddy currents achieve their maximum on the surface of the material and with the increased depth their attenuation and phase delay is observed. This phenomenon is called the skin effect $[1,2]$.

The basis for the standard eddy current testing of material is the movement of the probe over it. In the presence of a defect in given material, a change in coil impedance or induced voltage is recorded [2]. The ECT probe used in this study consists from two multi-turn coils: the exciting coil generating a time-varying electromagnetic field and sensing coil recording the resulting signal.

Sweep Frequency Response Analysis (SFRA) is reliable and sensitive method successfully used in the field of diagnosis of the transformer winding. For example, the move of the winding of its position or deflection of winding is possible to detect by this technique $[3,4]$.

The swept frequency eddy current technique connects the SFRA with eddy current method. The aim of this technique is to obtain a response to the change of the excitation signal in a wide frequency range and it is commonly used for measuring the coating thickness of conductive material. The authors propose to employ swept frequency

Department of Electromagnetic and Biomedical Engineering, Faculty of Electrical Engineering, University of Žilina, Žilina, Slovakia, andrea.stubendekova@fel.uniza.sk 


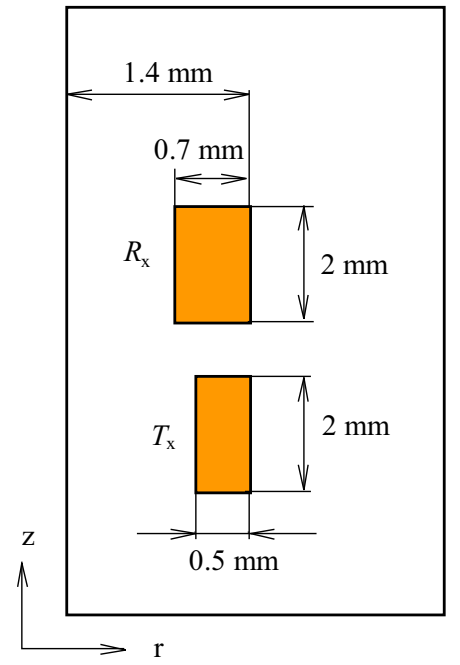

Fig. 1. Configuration of the eddy current air probe

excitation also for the detection and subsequently for the identification of defects $[5,6]$.

\section{CIRCUIT MODEL OF EDDY CURRENT AIR-CORE PROBE}

A suitable design of an ECT probe operating in a wide frequency range is required. The probe transfers information between the ECT instrument and investigated object and for this reason it is advantageous to know its characteristics to get good performance.

Eddy current air-core probe located in air was tested at first. The exciting coil $T_{x}$ was formed by 80 currentturns and the sensing coil $R_{x}$ by 140 turns. The coils have different internal diameters as shown in Fig. 1.

A high frequency voltage generator was used to drive the exiting coil. The input voltage $u_{1}$ and output voltage $u_{2}$ at terminals of the exciting and the receiving coils were recorded using the oscilloscope within the frequency range 7 to $20 \mathrm{MHz}$. The result of measurement shows the resonance approximately at $14 \mathrm{MHz}$.

The numerical model of the probe was created using a circuit model in the LTspice software at first, and subsequently it was verified in the COMSOL Multiphysics software. There is also a modern code Agros2D by which $2 \mathrm{D}$ calculations were verified. This simulation program is based on the fully adaptive higher-order finite element method [7].

The numerical model includes the measuring chain and the high-frequency model of the probe. Since the parameters of this chain influence the resulting frequency characteristics of the voltage transfer, their values are very important for creating the reliable model.

The results of 3D simulation show the resonance approximately at $13.5 \mathrm{MHz}$, see Fig. 2 .

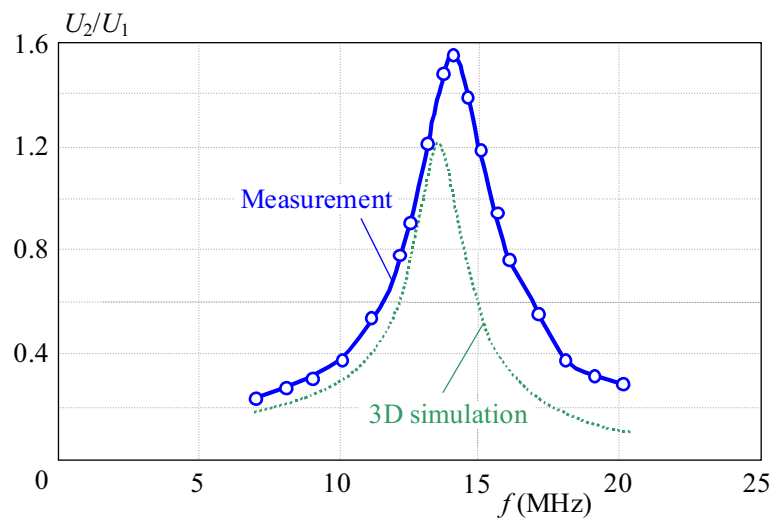

Fig. 2. The comparison of amplitude frequency characteristic of measurement and 3D simulation

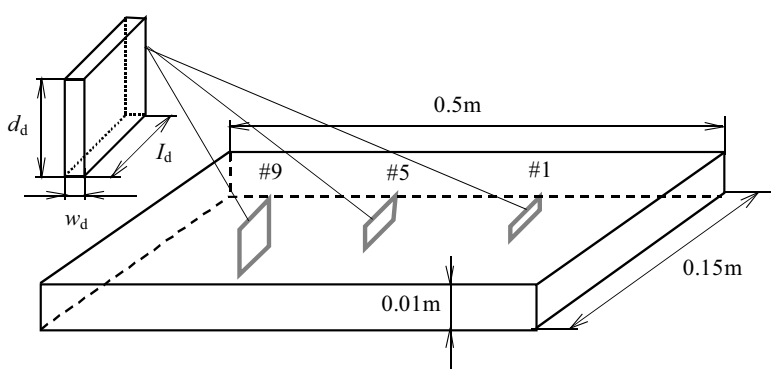

Fig. 3. The dimensions of plate specimen with artificial defects

The obtained results of experiment and numerical simulation of air probe itself provide similar frequency dependences so it can be concluded that the model provides realistic results. The differences are caused by several reasons, eg the number of the measurements or uncertainties of some input values of the equivalent circuit of the measurement chain. Further, the dimensions, configuration and values of parameters of the used coils are not entirely known.

\section{NON-DESTRUCTIVE TESTING OF CONDUCTIVE MATERIAL}

The purpose is to detect and estimate resolution of the defects in different depths. A plate made of austenitic steel SUS316L was evaluated in this study. The conductivity of material was $\gamma=1.4 \times 10^{6} \mathrm{~S} / \mathrm{m}$ and the relative permeability $\mu_{r}=1$. Three electro-discharge machined notches, ie non-conducting defects, with different depths $d_{d}=1.5$ and $9 \mathrm{~mm}$ were investigated. All defects had rectangular shape, the same thickness $w_{d}=0.25 \mathrm{~mm}$ and the length is $l_{d}=10 \mathrm{~mm}$. The configuration of inspected plate specimen is shown in Fig. 3

The measurement was made with the same eddy current air-core probe as in the previous case. The external diameter of the coils was the same $d_{\text {Txout }}=d_{\text {Rxout }}=$ $2.8 \mathrm{~mm}$ and the height of coils was $v=2 \mathrm{~mm}$. The internal diameter of the exciting coil was $d_{\text {Txin }}=1.8 \mathrm{~mm}$ and 


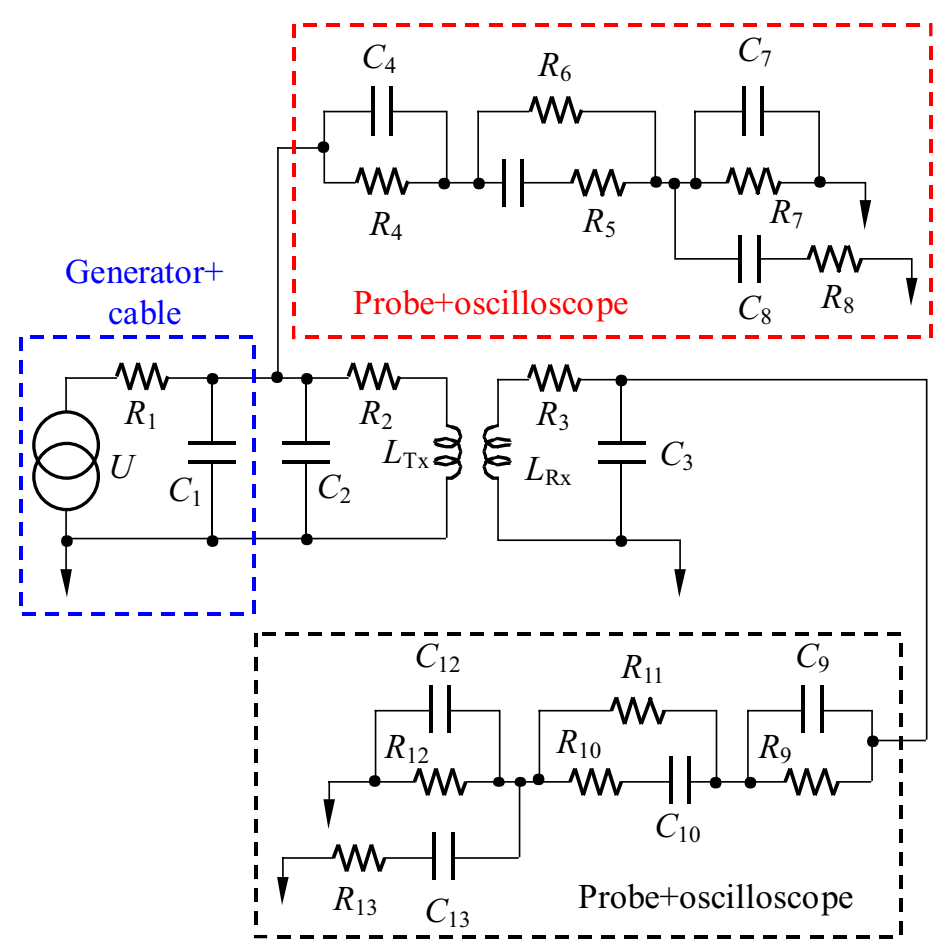

Fig. 4. Arrangement of the feeding circuit

of the sensing coil was $d_{R x i n}=1.4 \mathrm{~mm}$. The exciting coil was supplied with the harmonic current.

The probe was located perpendicularly to the surface of the material, directly over defects for particular inspections as shown in Fig. 3. The investigated material is positioned closer to exciting coil. The detailed scheme of the feeding circuit is depicted in Fig. 4.

The probe was distant by $h \approx 0.5 \mathrm{~mm}$ from the surface of the austenitic steel plate because of the probe encapsulation. The probe was fixed by a holder to achieve good accuracy of measurement results. The exciting frequency was changed in the range mentioned above and the ratio of output and input voltages $u_{2} / u_{1}$ were evaluated for different exciting frequencies.

The numerical model of inspection was created in 3D COMSOL Multiphysics software. Both equations (partial differential equation for magnetic field and ordinary differential equation for the circuit in Fig. 4 were solved in the hard-coupled formulation.

The field equation written in terms of magnetic vector potential $\overrightarrow{\mathcal{A}}$ is

$$
\Delta \overrightarrow{\mathcal{A}}-j \omega \mu_{0} \gamma \overrightarrow{\mathcal{A}}=-\mu_{0} \overrightarrow{\mathcal{J}}_{\text {ext }}
$$

where $\omega$ is the angular velocity, $\mu_{0}$ is the relative permeability of all parts in the system (the plate is made of austenitic steel), $\gamma$ is the electrical conductivity and $\overrightarrow{\mathcal{J}}_{\text {ext }}$ is the external current density. The distribution of the field quantities then provides the value of inductance and induced voltage for the circuit model.
The numerical simulations were carried out using the same configuration where the depth of defect was changed $d_{d}=1,5$ and $9 \mathrm{~mm}$. Therefore, they were performed for each defect separately. The frequency range was set to the same scope $f \in\langle 7 \mathrm{MHz}, 20 \mathrm{MHz}\rangle$ as in the measurements. The parameters of the model, its properties and the mesh were the same for the entire test. The mesh in the vicinity of the defect was finer to obtain more precise results. The distance between the probe and evaluated object was $h=0.1 \mathrm{~mm}$ for the numerical simulations.

The results of experiments and numerical simulations are shown in Figs. 5 and 6 . The amplitude frequency characteristic or the voltage-amplitude transfer $U_{2} / U_{1}$ is displayed. The experiments and numerical simulations show the similar results and in the both cases the differences between the signals of defects with various depths are very small. A better view of results and highlight of disparities between the signals are the reasons for which these dependences are displayed in a smaller range of frequencies, $i e$ in the resonance range. The signal causing the biggest deflection represents the result of detection of the deepest defect.

The resonance frequency in experiments was approximately $f_{R}=14 \mathrm{MHz}$ and the values of voltage-amplitude transfer were $U_{2} / U_{1}=1.48684 ; 1.4902$ and 1.5 for individual defects with a depth $d_{d}=1,5$ and $9 \mathrm{~mm}$, respectively.

The resonance frequency in numerical simulations was $f_{R}=13.5 \mathrm{MHz}$ and the values of voltage-amplitude transfer were $U_{2} / U_{1}=1.16121 ; 1.16284$ and 1.16341 for defects with a depth $d_{d}=1,5$ and $9 \mathrm{~mm}$, respectively. 


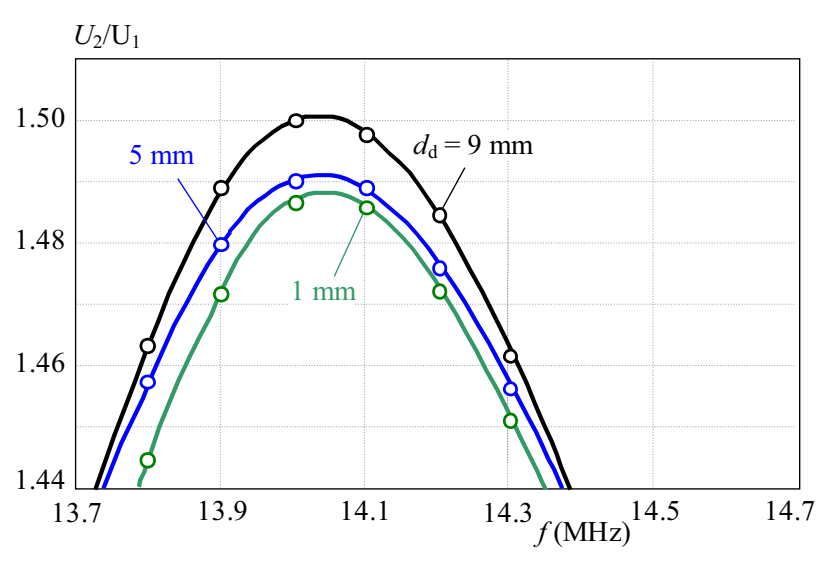

Fig. 5. Amplitude-frequency characteristics as measured at three different defects

Likely the geometry change of a defect causes a change of its capacitance, thereby it influences the resulting signal.

\section{CONCLUSION}

The connection of eddy current method with swept frequency technique represents efficient method for nondestructive investigation of various conductive objects containing the surface defects. The aim of this article was to develop a model of an air-core eddy current probe, which would be applicable for numerical simulations in a wide frequency range. LTspice and COMSOL Multiphysics software were used to develop the model. Subsequently, this model was used for numerical simulation of non-destructive inspection of an austenitic steel plate containing three surface defects of various depths.

Frequency characteristics of the probe transfer function were evaluated. The results of measurements and numerical simulations showed very similar behaviour. The frequency characteristics depend on a defect depth.

Based on the achieved results it can be concluded that the swept frequency technique is applicable in the field of non-destructive testing by eddy current and it has an extensive potential of employment regarding the requirements of modern diagnostic techniques.

\section{Acknowledgement}

This work was supported by the Slovak Research and Development Agency under the contracts No. APVV0349-10.

\section{REFERENCES}

[1] RAO, B. P. C.: Practical Eddy Current Testing, Alpha Science International Limited, 2007.

[2] STRAPACOVA, T.-JANOUSEK, L.-SMETANA, M.-CAPOVA, K.: Evaluation of Advanced Sensor Types Under Harmonic Excitation in ECT, Studies in Applied Electromagnet-

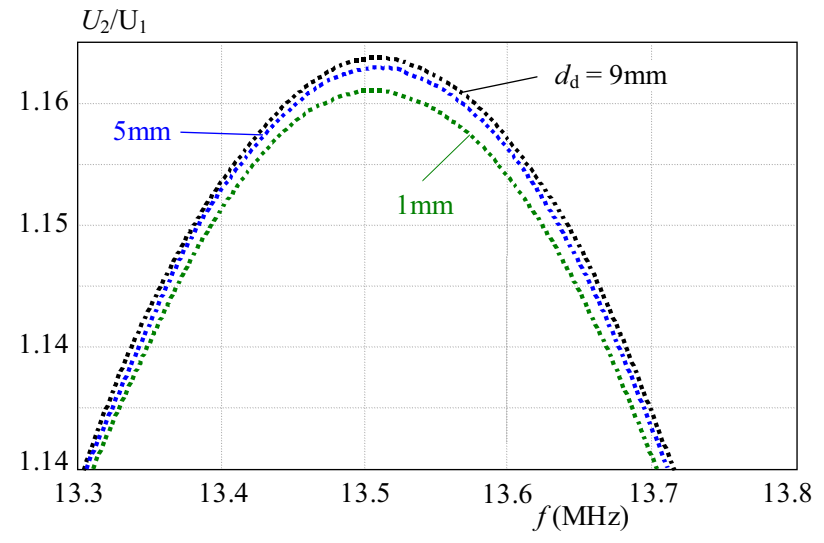

Fig. 6. Amplitude-frequency characteristics — numerical simulation of three different defects

ics and Mechanics, Electromagnetic Nondestructive Evaluation (XVI), vol. 38, 2014, pp. 9-15.

[3] PANDYA, A.-PAREKH, B. R. : Interpretation of Sweep Frequency Response Analysis (SFRA) Traces for the Open Circuit and Short Circuit Winding Fault Damages of the Power Transformer, International Journal of Electrical Power \& Energy Systems (Nov 2014), 890-896.

[4] Sweep Frequency Response Analysis Test, [online], [2014/12/8], http://www.electrical4u.com/ sweep-frequency-response-analysis-test-sfra-test/.

[5] STRAPACOVA, T.-CAPOVAK.-JANOUSEK, L.-SMETANA, M.: Defect Identification using Eddy Current Sweep Frequency Technique, Studies in Applied Electromagnetics and Mechanics, Electromagnetic Nondestructive Evaluation (XV), vol. 36, 2012, pp. 264-260.

[6] Swept frequency, [online], [2014/12/8], https://www.nde-ed.org/ EducationResources/ CommunityCollege/EddyCurrents/AdvancedTechniques/ sweptfrequency.htm.

[7] KARBAN, P.-MACH, F.-KUS, P.-PANEK, D.-DOLEZEL, I. : Numerical Solution of Coupled Problems Using Code Agros2D, Computing 95 No. 1 Supplement (2013), 381-408, DOI 10.1007/s00607-013-0294-4.

Received 10 January 2015

Andrea Štubendeková was born in Dolný Kubín. In 2013 she graduated (MSc) at the Department of Electromagnetic and Biomedical Engineering of the Faculty of Electrical Engineering at University of Zilina. She is $\mathrm{PhD}$ student in the said department and her thesis title is Non-destructive testing of conductive materials by eddy current method based on swept frequency.

Ladislav Janoušek was born in Michalovce. He graduated (master study) at the Department of Power Electrical Systems, Faculty of Electrical Engineering, University of Zilina in 1997. He continued with PhD. study at the same department in the field of electric machines and graduated in 2002. He became an associate professor in the field Theory of Electrical Engineering in 2007. Currently he works at the Department of Electromagnetic and Biomedical Engineering, Faculty of Electrical Engineering, University of Žilina. His research activities are focused on electromagnetic methods of non-destructive evaluation and influence of electromagnetic field on live organisms. 